[Aus dem hygienischen Institut der Kgl. Universität Berlin.]

(Leiter: Prof. C. Flügge.)

\title{
Über \\ die Beeinflussung der Arbeitsleistung am Ergographen durch längeren Aufenthalt in geschlossenem Raume.
}

Von

Stabsarzt Dr. Schuster,

kommandiert zum Institat.

Nachdem zahlreiche frühere Untersuchungen eine akute und deutliche gesundheitsschädliche Wirkung menschlicher Ausatmungsluft nicht hatten feststellen können (Hermans, Beu, Rauer, Formánek u. a.), lag es nahe, feinere Prüfungsmittel für den Nachweis einer solchen Wirkung heranzuziehen und speziell die Arbeitsleistung als Mabstab für eine ungünstige Beeinflussung des menschlichen Körpers durch wiedereingeatmete Exspirationsstoffe zu benutzen.

Schon im Laufe des Jahres 1911/12 hatte Konrich auf Veranlassung von Herrn Geheimrat Flügge eine Reihe von Untersuchungen darüber angestellt, ob durch den Aufenthalt in einem gut verschlossenen Raum und die dadurch bedingte Luftverschlechterung eine merk- und meBbare Herabsetzung der Arbeitsleistung am Ergographen verursacht werde. Er ging bei diesen Versuchen im allgemeinen in der Weise vor, daB eine oder mehrere Versuchspersonen mehrere Stunden in einem Versuchskasten, entweder demselben, den er bei seinen Ozonversuchen benutzte, oder einem gröBeren eingeschlossen wurden. Bei Beginn und am Ende des Versuchs wurde die Arbeitsleistung mittels des Mossoschen Ergographen in der von Dubois angegebenen Modifikation geprüft. Da bei diesen Versuchen teilweise auch Wärmestauung als schädliches Agens mit in Betracht kommen 
konnte, wurden - neben fortlaufenden Messungen der Lufttemperatur und Luftfeuchtigkeit im Versuchsraum - auch die Stirn- und Bluttemperatur der Versuchspersonen, sowie deren Blutdruck bestimmt.

Einen Teil der Konrichschen Versuchsergebnisse, die mir von ihm zu diesem Zweck freundlichst zur Verfügung gestellt wurden, habe ich in Tabelle I zusammengestellt. Es handelt sich um 34 Einzelversuche. Davon betrafen 21 einen Aufenthalt im geschlossenen Kasten ohne Luftzufuhr und ohne Zirkulation der Luft. Dabei wurde 8 mal Anstieg der Leistung, 1 mal Gleichbleiben, 12 mal Abnahme beobachtet; von letzteren Versuchen sind aber Nr. 15 bis $\mathbf{1 7}$ nicht einwandfrei, weil die Versuchsperson, vermutlich infolge bereits bestehender Ermüdung, abnorm geringe Anfangswerte aufwies. Bei Zirkulation der verunreinigten Kastenluft, ohne Zufuhr frischer Luft, ergaben 4 Versuche Anstieg, 4 Abnahme. Von Versuchen bei offener Tür des Kastens zeigten 3 Anstieg, 2 Abnahme.

Die Versuchsresultate waren mithin nicht eindeutig. Zum Teil lag dies wohl daran, daß die Kontrollversuche nicht zahlreich genug waren. Ferner war die Dauer der Versuche und die Luftrerunreinigung einige Male so weit getrieben, daB schon die $\mathrm{CO}_{2}$-Ansammlung toxische Wirkungen auszulösen vermochte (bis 5 Prozent!). Diesem Umstand war es wohl zuzuschreiben, daB in ein paar Fällen bei den Versuchspersonen sogar akute Störungen, ohnmachtähnliche Zustände, auftraten. Auch die Luftfeuchtigkeit und die Temperatur im Kasten erreichten in diesen Fällen allerdings eine solche Höhe, daß mit einer Gesundheitsstörung durch Wärmestauung gerechnet werden muB.

Eine kleine Reihe weiterer Versuche, auf deren Wiedergabe ich verzichte, brachte gleichfalls keine Klärung. Ganz regellose Abweichungen von der Norm zeigten die Zahlen für Stirn- und Bluttemperatur, sowie die Blutdruckmessungen.

Da Konrich aus äußeren Gründen nicht in der Lage war, die Versuche in größerer Reihe fortzusetzen, veranlaßte mich Herr Geheimrat Flügge zu Anfang des Sommers 1913, dieselben mit etwas anderer Versuchsanordnung wieder aufzunehmen. Namentlich sollten die Fehlerquellen, die sich im Laufe der Konrichschen Versuche ergeben hatten, nach Möglichkeit vermieden werden. Vor allem wollte ich Übertreibungen in der Verunreinigung der Luft und auch bezüglich der Temperatur und Feuchtigkeit vorbeugen. Ferner waren Fehler der Beobachtung am Ergographen auszuschalten.

Von verschiedenen Seiten ist schon darauf hingewiesen, dab man nur nach längerem sorgfältigen Training geeigneter, d. h. intelligenter und nicht nervöser oder zu temperamentvoller Versuchspersonen gleichmäßig ausfallende, verwertbare Ergographenkurven erhalten kann. Unbedingt 
Tabelle I.

\begin{tabular}{|c|c|c|c|c|c|c|c|c|}
\hline \multirow{2}{*}{ 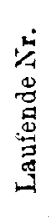 } & \multirow{2}{*}{ 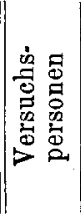 } & \multirow{2}{*}{ 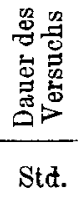 } & \multicolumn{2}{|c|}{$\begin{array}{l}\text { Kasten- } \\
\text { temperatur }\end{array}$} & \multirow{2}{*}{$\begin{array}{c}\mathrm{CO}_{2} \text { nach } \\
\text { Petten- } \\
\text { kofer, am } \\
\text { Ende des } \\
\text { Versuchs } \\
\%_{00}\end{array}$} & \multicolumn{2}{|c|}{$\begin{array}{l}\text { Ergographen- } \\
\text { arbeit }\end{array}$} & \multirow{2}{*}{ Bemerkungen } \\
\hline & & & $\begin{array}{l}\text { vor- } \\
\text { her }\end{array}$ & $\begin{array}{c}\text { nach- } \\
\text { her }\end{array}$ & & vorher & nachher & \\
\hline 1 & $\left\{\begin{array}{l}\mathrm{K}_{0} . \\
\mathrm{Ca} .\end{array}\right.$ & 2 & 18 & $20 \cdot 5$ & 20 & $\begin{array}{l}6.578 \\
5 \cdot 278\end{array}$ & $\begin{array}{l}6 \cdot 879 \\
5 \cdot 047\end{array}$ & $\begin{array}{l}\text { Kasten geschlossen, } \\
\text { ohne Ventilation }\end{array}$ \\
\hline 2 & $\left\{\begin{array}{l}\mathrm{Ko} o . \\
\mathrm{Ho} .\end{array}\right.$ & 2 & 20 & 22 & $20 \cdot 5$ & $\begin{array}{l}7 \cdot 384 \\
7 \cdot 247\end{array}$ & $\begin{array}{l}6 \cdot 988 \\
6 \cdot 578\end{array}$ & desgl. \\
\hline 3 & $\left\{\begin{array}{l}\text { Ko. } \\
\text { Ku. }\end{array}\right.$ & 2 & 20 & 23 & $20 \cdot 35$ & $\begin{array}{l}7 \cdot 067 \\
9 \cdot 077\end{array}$ & $\begin{array}{c}6 \cdot 23 \\
10.597\end{array}$ & $"$ \\
\hline 4 & Ko. & 2 & 20 & $22 \cdot 5$ & 20 & $7 \cdot 959$ & $7 \cdot 51$ & $\begin{array}{l}\text { Kasten geschlossen, } \\
\text { Luft zirkuliert }\end{array}$ \\
\hline 5 & $\left\{\begin{array}{l}\mathrm{Ko} . \\
\mathrm{Ku} .\end{array}\right.$ & 2 & 22 & $23 \cdot 5$ & $0 \cdot 06$ & $\begin{array}{r}8 \cdot 202 \\
10 \cdot 357\end{array}$ & $\begin{array}{l}9 \cdot 171 \\
9 \cdot 171\end{array}$ & Tür des Kastens offen \\
\hline 6 & $\left\{\begin{array}{l}\text { Ko. } \\
\text { Ti. }\end{array}\right.$ & $23 / 4$ & $16 \cdot 5$ & 18 & $24 \cdot 5$ & $\begin{array}{l}8 \cdot 5 \\
2 \cdot 7\end{array}$ & $\begin{array}{l}8 \cdot 6 \\
4 \cdot 9\end{array}$ & $\begin{array}{l}\text { Kasten geschlossen, } \\
\text { ohne Ventilation }\end{array}$ \\
\hline 7 & $\left\{\begin{array}{l}\text { Ko. } \\
\text { Ti. }\end{array}\right.$ & 2 & 17 & 19 & $18 \cdot 56$ & $\begin{array}{l}7 \cdot 5 \\
3 \cdot 4\end{array}$ & $\begin{array}{l}9 \cdot 1 \\
3 \cdot 9\end{array}$ & desgl. \\
\hline 8 & $\left\{\begin{array}{l}\text { Ko. } \\
\text { Ti. }\end{array}\right.$ & $2^{3} /_{4}$ & $17 \cdot 5$ & 20 & $28 \cdot 3$ & $\begin{array}{l}9 \cdot 1 \\
3 \cdot 8\end{array}$ & $\begin{array}{l}8 \cdot 0 \\
4 \cdot 1\end{array}$ & $"$ \\
\hline 9 & $\left\{\begin{array}{l}\text { Ko. } \\
\text { Bo. }\end{array}\right.$ & 2 & $19 \cdot 5$ & $22 \cdot 5$ & $7 \cdot 52$ & $\begin{array}{l}7 \cdot 9 \\
6 \cdot 4\end{array}$ & $\begin{array}{l}8 \cdot 0 \\
7 \cdot 0\end{array}$ & $\begin{array}{l}\text { Kasten geschlossen, } \\
\text { Luft zirkuliert }\end{array}$ \\
\hline 10 & Ca. & 2 & 17 & $19 \cdot 5$ & 21 & $5 \cdot 3$ & $5 \cdot 2$ & $\begin{array}{l}\text { Kasten geschlossen, } \\
\text { ohne Ventilation }\end{array}$ \\
\hline 11 & $\left\{\begin{array}{l}\text { Ko. } \\
\text { Bo. }\end{array}\right.$ & 2 & $20 \cdot 5$ & 23 & $10 \cdot 1$ & $\begin{array}{l}8 \cdot 1 \\
6 \cdot 7\end{array}$ & $\begin{array}{l}7 \cdot 8 \\
7 \cdot 2\end{array}$ & $\begin{array}{l}\text { Kasten geschlossen, } \\
\text { Luft zirkuliert }\end{array}$ \\
\hline 12 & $\left\{\begin{array}{l}\mathrm{Ko} . \\
\mathrm{Bo.} \\
\mathrm{Ca} .\end{array}\right.$ & 2 & 19 & $22 \cdot 5$ & $50 \cdot 74$ & $\begin{array}{l}7 \cdot 1 \\
8 \cdot 4 \\
5 \cdot 1\end{array}$ & $\begin{array}{l}7 \cdot 2 \\
7 \cdot 1 \\
5 \cdot 1\end{array}$ & $\begin{array}{l}\text { Kasten geschlossen, } \\
\text { ohne Ventilatiou }\end{array}$ \\
\hline 13 & $\left\{\begin{array}{l}\text { Ko. } \\
\text { Ho. } \\
\text { Ca. }\end{array}\right.$ & 2 & $21 \cdot 5$ & $23 \cdot 5$ & $17 \cdot 2(?)$ & $\begin{array}{l}8 \cdot 814 \\
7 \cdot 123 \\
5 \cdot 015\end{array}$ & $\begin{array}{l}7 \cdot 075 \\
7 \cdot 571 \\
4 \cdot 723\end{array}$ & $\begin{array}{l}\text { Kasten geschlossen, } \\
\text { Luft zirknliert }\end{array}$ \\
\hline 14 & $\left\{\begin{array}{l}\mathrm{Ko} . \\
\mathrm{Ho} . \\
\mathrm{Ca} .\end{array}\right.$ & 2 & 22 & $23 \cdot 5$ & 0.05 & $\begin{array}{l}8 \cdot 033 \\
7 \cdot 168 \\
4 \cdot 969\end{array}$ & $\begin{array}{l}8 \cdot 883 \\
6 \cdot 781 \\
5 \cdot 175\end{array}$ & $\begin{array}{c}\text { Tür des Kastens } \\
\text { offen }\end{array}$ \\
\hline 15 & Ко. & 3 & - & - & $12 \cdot 9$ & $5 \cdot 2$ & $4 \cdot 6$ & $\begin{array}{l}\text { Kasten geschlossen, } \\
\text { ohne Ventilation }\end{array}$ \\
\hline 16 & Ko. & 4 & - & - & $16 \cdot 7$ & $6 \cdot 9$ & $6 \cdot 3$ & desgl. \\
\hline 17 & Ko. & 3 & - & - & $16 \cdot 6$ & $5 \cdot 6$ & $4 \cdot 9$ & $"$ \\
\hline 18 & Ko. & 3 & - & - & 8.5 & $7 \cdot 5$ & $6 \cdot 0$ & $"$ \\
\hline 19 & Ko. & $21 / 4$ & - & - & - & $7 \cdot 183$ & 7.014 & $\begin{array}{l}\text { Versuchsperson liest wäh- } \\
\text { rend des Versuchs u. miBt } \\
\text { nur Ergogr.-Arbeit }\end{array}$ \\
\hline
\end{tabular}


sollten auch die Versuchspersonen nicht wie in den Konrichschen Versuchen mit den zum Versuch gehörigen Arbeiten, die an sich eine körperliche und geistige Anstrengung darstellten, belastet, sondern lediglich zu der Arbeit am Ergographen herangezogen werden.

Für die Versuche wurde ausschlieblich ein etwa 3.8 ${ }^{\mathrm{cbm}}$ fassender, aus Holz und groBen Glasscheiben bestehender Versuchskasten benutzt, dessen Tür sich luftdicht verschließen lieb. Auf der der Tür gegenüberliegenden Schmalseite befindet sich unten und oben je eine Öfnung von $15^{\mathrm{cm}}$ Durchmesser, von denen die obere durch ein Rohr mit einem Holzkasten in Verbindung steht, der einen elektrischen Ventilator enthält. Von der unteren Öffnung führt ein anderes Rohr direkt in einen Ventilationsschacht des Hauses. Durch mehrere, in diese Röhren eingebaute Schieber kann erreicht werden, daB die Kastenluft entweder stagniert, zirkuliert oder entfernt und durch frische Luft ersetzt wird. Durch einen außen an dem Kasten angebrachten Vorhang wird es der im Innern befindlichen Versuchsperson unmöglich gemacht, die sich auBen abspielenden Vorgänge zu beobachten; da der Ventilator bei allen Versuchen gleichmäBig im Gang gehalten wurde, konnte die Versuchsperson auch durch das Geräusch nicht darauf' aufmerksam gemacht werden, daB eine Änderung in bezug auf Luftzufuhr, Luftabschluß oder Luftbewegung vorgenommen war. Durch Gummistopfen verschlieBbare kleine Öfnungen ermöglichen die Entnahme von Luftproben von außen her. Im Innern des Kastens befindet sich ein kleiner Tisch, auf welchem der Ergograph angeschraubt ist, ein Stuhl und in einer Ecke eine kleine Konsole zur Aufnahme der zur Messung der Stirntemperatur erforderlichen Instrumente.

Zur Prüfung der Arbeitsleistung wurde der Ergograph von Dubois beibehalten, bei welchem die Leistung des rechten Zeigefingers geprüft wird. Die Belastung betrug $5 \mathrm{~kg}$. Um eine, wenn auch vielleicht unbeabsichtigte Mitarbeit anderer Muskelgruppen nach Möglichkeit auszuschließen, wurde außer dem Handgelenk auch noch der Oberarm bei rechtwinklig gebeugtem Arm dicht über dem Ellbogen durch einen an dem Tisch angebrachten breiten Lederriemen fixiert.

Auf die Notwendigkeit, bei der Prüfung der Arbeitsleistung durch den Ergographen nur geeignete und sorgfältig trainierte Personen zu verwenden, ist bereits oben hingewiesen. Neuerdings hat Berliner gegen die Brauchbarkeit des Ergographen zur Messung der Höchstleistung eingewendet, daB die Hubhöhe zu begrenzt ist, und bei impulsiver Arbeit das Gewicht über die maximale Hubhöhe hinausgeschleudert wird. Diese Fehlerquelle wird man doch wohl durch eine der Kraft der betreffenden Versuchsperson entsprechende Steigerung der Belastung vermeiden können. Der von Berliner verwandte Weilersche "Arbeitsschreiber", ein Instru- 
ment, bei welchem die Leistung eines Dynamometers sich auf einer automatisch weiter rückenden Papierscheibe aufzeichnet, hat allerdings wohl den Vorzug, daB er leicht transportabel ist, aber, wie Berliner selbst zugibt, auch groBe Nachteile. Diese bestehen vor allem darin, daB die Eichung des Apparates eine sehr ungenaue ist, weil er zu viel Reibung und dadurçh eine zu große Trägheit hat.

Die Verwendung der Fuß-Hantel-Methode Weichardts, deren praktische Verwertbarkeit von verschiedenen Seiten angezweifelt wird, verbot sich schon mit Rücksicht auf den beschränkten Raum im Versuchskasten.

Messungen der Stirntemperatur wurden mit der thermoelektrischen Methode vorgenommen, wie sie von Reichenbach und Heymann näher beschrieben worden ist. Die Temperatur wurde jedesmal unmittelbar vor und nach der Ergographenarbeit, und zwar stets auf derselben Hautstelle, festgestellt. Die Messungen ergaben aber auch bei meinen Versuchen so wenig eindeutige Resultate, daB ich auf deren Wiedergabe verzichte.

Die gröBte Anzahl der Versuche sind an einem Assistenten des Instituts (Fi.) angestellt, der sich hierzu in liebenswürdiger Weise bereit erklärt hatte; auBerdem wurden noch 2 Institutsdiener (E. und Fr.) zu den Versuchen herangezogen. Um zunächst einen Überblick über die normale Ergographenleistung der Versuchspersonen zu gewinnen, wurden dieselben vor Beginn der eigentlichen Versuche längere Zeit hindurch einem Training, am Ergographen unterworfen. Da die eigentliche Versuchsdaner im allgemeinen $2^{1} / 2$ Stunden nicht überschreiten sollte, wurde anch bei diesen Vorversuchen die Ergographenleistung täglich zweimal mit etwa $21 / 2$ stündigem Zwischenraum möglichst immer zur gleichen Tageszeit gemessen. Um eine etwaige - vielleicht auch psychische - Beeinflussung durch den bloßen Aufenthalt in dem geschlossenen Kasten bei den späteren Versuchen mit Sicherheit ausschalten zu können, wurden diese vorhergehenden Prüfungen ebenfalls sämtlich teils im offenen, teils im geschlossenen Kasten vorgenommen. Die täglichen Übungen am Ergographen wurden von den drei Versuchspersonen während der eigentlichen Untersuchungen auch an den versuchsfreien Tagen fortgesetzt.

Bei den ersten Vorversuchen erhielt ich nach einiger Zeit trotz der zunehmenden Ubung geringere Werte, die erst wieder normal wurden, nachdem der Apparat auseinander genommen und gereinigt worden war. Offenbar ist es daher unbedingt erforderlich, vor-jedesmaligem Gebrauch sämtliche gleitenden Teile des Apparates gut zu reinigen und einzuölen, weil er sonst bei längerer Benutzung zu schwer läuft und nur aus diesem Grunde zu niedrige Werte ergibt.

Bei den folgenden eigentlichen Versuchen wurden nachstehende Punkte immer berücksichtigt: 
Am Morgen vor Beginn des Versuchs durfte die Versuchsperson keine oder wenigstens keine schwerere Arbeit verrichtet haben, eventuell muBte sie einige Zeit vorher ruhen. Angestellt wurde ein Versuch nur, wenn die betreffende Versuchsperson zu Beginn des Versuches eine normale, ihren sonstigen Kurven entsprechende Ergographenleistung zeigte. Während der ganzen Versuchsdauer durfte die Versuchsperson irgendwelche körperliche und anstrengende geistige Arbeit nicht verrichten, bescbäftigte sich vielmehr nur mit Lesen. Im übrigen wurden ihre sonstigen Lebensgewohnheiten (Frühstück usw.) nicht geändert. Die Versuche wurden stets nur so lange ausgedehnt, daB Störungen des Allgemeinbefindens irgendwelcher Art nicht zu befürchten waren; solche sind auch nie aufgetreten.

Tabelle II.

\begin{tabular}{|c|c|c|c|c|c|c|c|c|c|c|c|}
\hline \multirow{2}{*}{ 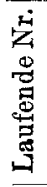 } & \multirow{2}{*}{ 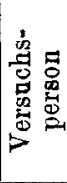 } & \multirow{2}{*}{ 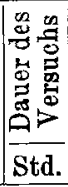 } & \multicolumn{2}{|c|}{$\begin{array}{l}\text { Zimmer- } \\
\text { temperatur }\end{array}$} & \multicolumn{2}{|c|}{$\begin{array}{c}\text { Kasten- } \\
\text { temperatur }\end{array}$} & \multicolumn{2}{|c|}{$\begin{array}{c}\mathrm{CO}_{2} \text { nach } \\
\text { Pettenkofer } \\
\% \%\end{array}$} & \multicolumn{2}{|c|}{\begin{tabular}{|} 
Ergographen- \\
arbeit \\
mkg
\end{tabular}} & \multirow[t]{2}{*}{ Bemerkungen } \\
\hline & & & $\begin{array}{l}\text { vor- } \\
\text { her }\end{array}$ & $\begin{array}{c}\text { nach- } \\
\text { her }\end{array}$ & $\begin{array}{l}\text { vor- } \\
\text { her }\end{array}$ & $\begin{array}{c}\text { nach- } \\
\text { her }\end{array}$ & $\begin{array}{l}\text { vor- } \\
\text { her }\end{array}$ & \begin{tabular}{|l} 
nach- \\
her
\end{tabular} & $\begin{array}{l}\text { vor- } \\
\text { her }\end{array}$ & $\begin{array}{l}\text { nach- } \\
\text { her }\end{array}$ & \\
\hline$\overline{1}$ & Fi. & $21 / 6$ & 18 & 18 & 19 & 21 & $1 \cdot 3$ & 8 & $9 \cdot 42$ & $10 \cdot 34$ & ohne V \\
\hline 2 & Fi. & & 19 & $19 \cdot 25$ & 20 & $22 \cdot 5$ & $0 \cdot 9$ & $9 \cdot 8$ & $10 \cdot 50$ & 70 & \\
\hline 3 & $\mathrm{Fi}$ & $2^{1}$ & 20.5 & 20.5 & $21 \cdot 5$ & $23 \cdot 5$ & 0.6 & $7 \cdot 2$ & 10.42 & 33 & $\begin{array}{l}\text { Kasten gest } \\
\text { Luft zirl }\end{array}$ \\
\hline 4 & Fi. & $2 \%$ & 23 & $23 \cdot 5$ & 23 & $24 \cdot 5$ & 0.56 & 0.62 & 0.42 & 13 & $\begin{array}{r}\text { Kasten of } \\
\text { mit Ventil }\end{array}$ \\
\hline 5 & Fi. & $1 / 2$ & $23 \cdot 5$ & 24 & $23 \cdot 5$ & 25 & 0.54 & 0.57 & $10 \cdot 64$ & 55 & \\
\hline 6 & Fi. & 011 & 20 & 20 & 21 & 23 & 0.55 & $10 \cdot 56$ & $13 \cdot 41$ & $14 \cdot 97$ & $\begin{array}{c}\text { Kasten ge } \\
\text { ohne Ve }\end{array}$ \\
\hline 7 & Fi. & & 16 & 16 & 17 & $19 \cdot 25$ & 0.55 & $7 \cdot 67$ & $13 \cdot 50$ & 14.92 & des \\
\hline 8 & Fi. & & 20 & $20 \cdot 25$ & 21 & 24 & 0.39 & .68 & $10 \cdot 58$ & $11 \cdot 40$ & $"$ \\
\hline 9 & Fr. & $21 / 2$ & $20 \cdot 75$ & $20 \cdot 75$ & 21 & $23 \cdot 5$ & 0.47 & $7 \cdot 52$ & $7 \cdot 835$ & $8 \cdot 36$ & " \\
\hline 0 & Fi. & $21 / 2$ & 20 & 20 & $20 \cdot 5$ & 23 & 0.56 & .13 & $11 \cdot 335$ & 13.115 & $"$ \\
\hline 11 & E. & $2^{1 / 4}$ & 16.5 & 17 & 18 & 21 & $0 \cdot 49$ & $\cdot 17$ & $6 \cdot 87$ & $7 \cdot 535$ & $"$ \\
\hline 12 & Fr. & $21 / 4$ & $17 \cdot 5$ & $17 \cdot 5$ & $18 \cdot 5$ & 21 & 0.57 & $8 \cdot 86$ & $8 \cdot 17$ & $8 \cdot 86$ & $n$ \\
\hline 3 & Fi. & & 18 & $18 \cdot 5$ & 19 & 21 & 0.56 & 0.71 & $11 \cdot 69$ & $13 \cdot 05$ & $\begin{array}{l}\text { Kasten gesc } \\
\text { mit Venti }\end{array}$ \\
\hline 4 & E. & $1 / 2$ & $17 \cdot 5$ & $17 \cdot 75$ & 18 & 21 & 0.61 & .76 & $8 \cdot 195$ & $8 \cdot 675$ & $\begin{array}{l}\text { Kasten ges } \\
\text { ohne Ven }\end{array}$ \\
\hline 15 & $\mathrm{Fr}$ & & 1 & 18 & 19 & $21 \cdot 25$ & 0.51 & .87 & $7 \cdot 705$ & $8 \cdot 250$ & $\begin{array}{l}\text { Kasten gess } \\
\text { Luft zirk }\end{array}$ \\
\hline 6 & Fi. & & 17.25 & $17 \cdot 5$ & 18.5 & $21 \cdot 25$ & 0.53 & $7 \cdot 62$ & $11 \cdot 665$ & 30 & desg \\
\hline 7 & Fr. & 21 & 21 & 21 & 21.5 & 23 & 0.50 & 0.73 & $9 \cdot 49$ & 10.41 & $\begin{array}{l}\text { Kasten ge: } \\
\text { mit Ven }\end{array}$ \\
\hline 8 & Fi. & $21 / 2$ & $20 \cdot 75$ & 21 & 21.25 & 22.5 & 0.53 & 0.61 & $11 \cdot 305$ & $12 \cdot 05$ & $\begin{array}{l}\text { Kasten offen, } \\
\text { ohne Ventilation }\end{array}$ \\
\hline$y$ & Fr. & $21 / 2$ & 20.5 & $20 \cdot 5$ & $21 \cdot 25$ & 22 & 0.51 & $0 \cdot 59$ & $10 \cdot 93$ & 11.40 & desgl. \\
\hline 20 & E. & $21 / 2$ & $18 \cdot 25$ & 18.25 & 19 & 20.5 & 0.43 & 0.52 & $9 \cdot 65$ & $9 \cdot 85$ & $n$ \\
\hline 1 & E. & $2^{1} / 2$ & $16 \cdot 5$ & 16.75 & $18 \cdot 5$ & 21 & 0.46 & 7.84 & 10.24 & 10.94 & $\begin{array}{l}\text { Kasten geschlo } \\
\text { Luft zirkulie }\end{array}$ \\
\hline
\end{tabular}


Die hauptsächlichsten zahlenmäßigen Resultate sämtlicher Versuche rrgeben sich aus Tabelle II. Bei der gröBeren Anzahl der Versuche blieb ver Kasten während der ganzen Versuchsdauer geschlossen ohne jegliche Ventilation. Bei den Kontrollversuchen wurde die Luft bei geschlossenem Kasten durch entsprechende Schieberstellung entweder in Zirkulation gelalten oder durch frische Luft ersetzt, oder es blieb die Tür des Kastens, welche die Hälfte des Querschnittes des Kastens einnahm und also im Verbältnis zum Kubikraum des Kastens eine sehr große Offnung darstellte, während des Versuches geöffnet. Mit Hilfe des elektrischen Ventilators konnten in der Minute etwa $3^{\mathrm{cbm}}$ Luft in den Kasten hineinbefördert werden, so daB ein auBerordentlich starker Ventilationseffekt zu erzielen war. Bei zwei Versuchen wurde bei geöffneter Tür außerdem noch ventiliert, und so eine intensive Zuglüftung hergestellt.

Die Messungen der Stirntemperatur haben niemals Steigerungen über $1^{0}$ hinaus während der ganzen Versuchsdauer ergeben. Es ist demnach bei keinem der Versuche Wärmestauung aufgetreten, die nach den Untersuchungsergebnissen Pauls objektiv erst dann nachweisbar ist, wenn der Ausschlag an der Stirn bei der Messung mit Thermoelementen mehr als $1^{0}$ beträgt.

Bei den vorhergehenden Trainingsübungen, bei denen die Versuchspersonen in der Zeit zwischen den beiden Messungen ihrer gewöhnlichen Beschäftigung nachgingen, war in der Regel bei der zweiten Messung die Ergographenleistung entweder nur wenig $(0.5$ bis höchstens $0.75 \mathrm{mkg})$ gesteigert, oder es war, namentlich öfters bei den beiden Dienern, eine Herabsetzung der Arbeitsleistung zu verzeichnen gewesen. Im Gegensatz hierzu hat sich, wie aus der Tabelle II hervorgeht, bei kein em der Kastenversuche eine Herabsetzung, vielmehr immer eine mitunter recht erhebliche Steigerung der Ergographenleistung (in einzelnen Fällen bis $1.5 \mathrm{mkg}$ und mehr) feststellen lassen. Der ruhige Aufenthalt im Kasten hat daher stets im Sinne einer Erholung gewirkt. Was nun aber als bedeutungsvolles Ergebnis hervortritt, das ist die Tatsache, daß es ganz gleichgültig war, ob die Versuchsperson in verunreinigter oder in reiner Luft sich aufhielt. Die Ausschläge im Sinne einer Erholung und Besserung der Leistungen waren bei allen Versuchen ungefähr die gleichen. Die mittlere Zunahme der Leistungen betrug bei den 10 Versuchen im geschlossenen Kasten ohne Luftbewegung im Mittel $=1.0$; in 4 Versuchen bei zirkulierender Luft $=\mathbf{0 . 9 4}$; in 5 Versuchen bei offenem Kasten oder Ventilation $=\mathbf{0 . 7 3}$. Nur bei den 2 Versuchen mit Ventilation und gleichzeitig offener Tür, wo auf die Personen ständig ein fühlbarer Luftstrom einwirkte, sind besonders hohe Zunahmen $(=\mathbf{1} \cdot 8)$ notiert, die aber wegen der geringen Zahl der Versuche nicht sicher auf diese fühlbaren Ströme zu beziehen 
sind und außerdem in einzelnen Versuchen ohne alle Luftzufuhr auch erreicht werden (Nr. 10). - Dabei war die Luftverunreinigung in den Versuchen im geschlossenen Kasten eine recht erhebliche. Der Kohlensäuregehalt der Luft erreichte im Mittel von 15 Versuchen 8.3 p. m., in einem Versuch $10.6 \mathrm{p} . \mathrm{m}$. Das sind Zahlen, die im praktischen Leben kaum je, selbst nicht in eng besetzten Schlafräumen gefunden werden. Man lann also sagen, daß die Luftverunreinigung durch Ausatmungsluft so hochgradig war, wie es überhaupt unter praktischen VerhäItnissen möglich ist. Dagegen zeigten die Versuche mit Ventilation oder offener Tür kaum einen merkbaren Anstieg des $\mathrm{CO}_{2}$-Gehalts. Und trotzdem keine Differenz der Ergographenleistung und, wie noch ausdrücklich hervorgehoben sei, auch des gesamten subjektiven Befindens der Versuchspersonen!

Es ergibt sich also aus diesen Versuchen, daB durch einen längeren bloBen Aufenthalt in einem geschlossenen Raume und durch die dabei zustande kommende starke Häufung von Exspirationsprodulten eine mittelst der Arbeitsleistung am Ergographen meBbare ungünstige Beeinflussung des lörperlichen Verhaltens nicht beobachtet werden konnte.

\section{Literatar-Verzeichnis.}

Berliner, Zeitschrift f. Balneologie. VI. Jahrg. S. 246.

Konrich, Diese Zeitschrift. Bd: LXXIII. S. 443.

Paul, Ebenda. Bd. XLIX. S. 405.

Reichenbach u. Heymann, Ebenda. Bd. LVII. S. 1.

Weichardt, Ebenda. Bu. LIX. S. 337. 